\title{
Evaluación de la integridad y permeabilidad de guantes de procedimientos utilizados en la clínica dental
}

\author{
Celso Leite De Miranda, ${ }^{1}$ \\ Felipe Vilasboas Rodrigues, ${ }^{2}$ \\ Janaina Emanuela Damasceno, ${ }^{3}$ \\ Carla Figueiredo Brandão, ${ }^{4}$ \\ Francisco Xavier Paranhos Coêlho Simões, ${ }^{4}$
}

\section{Resumen}

Objetivo: Este estudio tuvo como objetivo evaluar la integridad y la permeabilidad de los guantes de los procedimientos utilizados en el cuidado dental. Material y métodos: se evaluaron 100 guantes de dos marcas Unigloves e Descarpack, divididos de la siguiente manera: Grupo 1: guantes blancos; Grupo 2: rosa; Grupo 3: negro; Grupo 4: azul; Grupo 5: Transparente. Todos analizados visualmente uno por uno en las imperfecciones, los agujeros se inflaron con $700 \mathrm{ml}$ de agua teñida con violeta de genciana al $1 \%$ y se colgaron con una altura promedio de 1 metro de suelo a temperatura ambiente. Fueron evaluados por 2 horas, pero cada 30 minutos fueron inspeccionados por posible permeabilidad. Los datos se tabularon y analizaron utilizando la prueba $\mathrm{Chi}^{2}$ con $\mathrm{p}<0.05$. Resultados: durante el período de 30 y 60 minutos, los guantes no cambiaron en relación con la humedad y el goteo. En cuanto al grado de permeabilidad, no hubo diferencia estadística entre los grupos evaluados. Conclusión: Por lo tanto, podemos concluir que los diferentes colores o materiales de fabricación de los guantes de procedimiento no interfieren con su calidad.

Palabra clave: Guantes quirúrgicos, Contención de riesgos biológicos, Odontología.

${ }^{1}$ DDS. Dentistry course, School of Medicine and Public Health (EBMSP).

${ }^{2}$ DDS. Dentistry course, School of Medicine and Public Health (EBMSP)

${ }^{3}$ DDS. MSc. PhD student. Dentistry course, Piracicaba Dental School - University of Campinas (FOP/UNICAMP).

${ }^{4}$ DDS. MSc. PhD. Professor in School of Medicine and Public Health (EBMSP).

${ }^{5}$ DDS. MSc. PhD. Professor in School of Medicine and Public Health (EBMSP). 
Artigo original

\section{Avaliação da integridade e permeabilidade de luvas de procedimentos utilizados na clínica odontológica}

\section{Resumo}

Objetivo: Este estudo teve como objetivo avaliar a integridade e permeabilidade das luvas dos procedimentos utilizados no atendimento odontológico. Material e Métodos: Foram avaliadas 100 luvas de duas marcas Unigloves e Descarpack, divididas da seguinte forma: Grupo 1: luvas brancas; Grupo 2: rosa; Grupo 3: preto; Grupo 4: azul; Grupo 5: Transparente. Todos analisados visualmente um por um sobre imperfeições, os buracos foram inflados com $700 \mathrm{ml}$ de água manchada com violeta de genciana a $1 \%$ e pendurados com uma altura média de 1 metro de solo em temperatura ambiente.
Eles foram avaliados por 2 horas, porém a cada 30 minutos foram inspecionados para observar possível permeabilidade. Os dados foram tabulados e analisados por teste Qui ${ }^{2}$ com $p<0,05$. Resultados: durante o período de 30 e 60 minutos, as luvas não apresentaram alteração em relação à umidade e ao gotejamento. Quanto ao grau de permeabilidade, não houve diferença estatística entre os grupos avaliados. Conclusão: Assim, podemos concluir que as diferentes cores ou materiais de fabricação das luvas de procedimento não interferem em sua qualidade.

Palavras-chave: Luvas cirúrgicas, Contenção de riscos biológicos, Odontologia.

Original article

\section{Evaluation of glove integrity and permeability of procedures used in dentistry clinic}

\begin{abstract}
Objective: This study aimed to assess the integrity and permeability of gloves from procedures used in dental care. Material and Methods: 100 gloves of two brands were evaluated, divided asfollows: Group 1: white gloves; Group 2: pink; Group 3: black; Group 4: blue; Group 5: Transparent. All analyzed visually one by one on imperfections, the holes were inflated with $700 \mathrm{ml}$ of water stained with $1 \%$ gentian violet and hung with an average height of 1 meter of soil at room temperature. They were evaluated
\end{abstract}

for 2 hours, but every 30 minutes they were inspected for possible permeability. The data were tabulated and analyzed using the Qui ${ }^{2}$ test with $p<0.05$. Results: during the period of 30 and 60 minutes, the gloves did not change in relation to humidity and dripping. As for the degree of permeability, there was no statistical difference between the groups evaluated. Conclusion: Thus, we can conclude that the different colors or materials of manufacture of the procedure gloves do not interfere with their quality.

Keyword: Surgical gloves, Containment of biological risks, Dentistry 


\section{Introducción}

Una de las preocupaciones observadas por los pacientes es si el profesional adoptó, como control de infecciones, el uso de guantes de procedimiento, ya que el principal medio de prevención contra la transmisión de enfermedades infecciosas es el uso adecuado de Equipos de Protección Individual (EPI). ${ }^{1}$ En su actividad, el cirujano dental expone a sus pacientes, su equipo, él mismo y su familia a un universo microbiano altamente agresivo. ${ }^{1,2}$

Los guantes tienen tres funciones principales: reducen la posibilidad de que el profesional se infecte con patógenos presentes en la sangre y / o saliva de los pacientes; reducen las posibilidades de que el dentista transmita microorganismos de la microbiota de sus manos a los pacientes y reducen las posibilidades de transmitir microorganismos orales de un paciente a otro. $^{2,3}$

Desde un simple examen clínico hasta cirugías complejas, el equipo dental debe conocer y adoptar estándares de bioseguridad. ${ }^{4}$ Sin embargo, para que los guantes quirúrgicos $\mathrm{y}$ de procedimiento actúen, de hecho, como barreras fundamentales en la prevención de infecciones, por ejemplo: hepatitis $B$ y $\mathrm{VIH}$, mitigando el riesgo de infección cruzada, es necesario analizar y mantener la integridad de estos guantes durante Realización de los trámites.

La tasa de contaminación puede reducirse significativamente mediante la educación en relación al uso de EPP por parte de los profesionales de la salud, ya que el riesgo de esta exposición está directamente influenciado por factores ambientales, como la manipulación de instrumentos cortantes o equipos contaminados. La posibilidad de contaminación aumenta cuando hay una exposición más prolongada y frecuente del profesional a la sangre del paciente infectado. ${ }^{4}$

Se ha demostrado que la saliva, un fluido corporal de contacto constante e inevitable con el dentista, es el segundo vehículo más importante de enfermedades infecciosas, lo que favorece el uso de barreras mecánicas por parte de los profesionales de la salud. ${ }^{4}$

En materia de seguridad, se creó la creencia de que los guantes no tendrían la función de protección frente a los microorganismos, dado que, para que desempeñen adecuadamente su función, es fundamental que tengan buena calidad y no presenten una solución de continuidad que permita la difusión. de estos patógenos. ${ }^{3}$

El presente estudio tuvo como objetivo evaluar la permeabilidad e integridad de los guantes utilizados en los procedimientos utilizados en la atención al paciente en el consultorio dental.

\section{Materiales y métodos}

Se utilizaron guantes de procedimientos ambidiestros en diferentes colores y dos marcas Unigloves (Curitiba, PR - Brasil) y Descarpack (São Paulo, SP - Brasil). El investigador recogió 20 guantes elegidos al azar de cada caja, resultando en 5 grupos. La elección de las marcas se debió a la mayor cantidad de colores existentes 
en la tienda de productos dentales, siendo estos la marca Unigloves (Curitiba, PR Brasil), con látex en los colores: blanco y rosa; nitrilo en colores: negro y azul; y la marca Descarpack (São Paulo, SP - Brasil) y Vinyl, que es transparente. Todos los guantes se usaron dentro de la fecha de vencimiento.

Seprobaron 100 guantes de procedimientos no estériles talla " $\mathrm{M}$ ", distribuidos de la siguiente manera: Grupo 1 - Blanco (Lote $\mathrm{Z} 0265216 \mathrm{H}$ con fecha de fabricación agosto / 2016) - Unigloves (Curitiba, PR - Brasil); Grupo 2 - Rosa (lote Z08232017F con fecha de fabricación junio / 2017) - Unigloves (Curitiba, PR - Brasil); Grupo 3 - Negro (lote Z0922817E con fecha de fabricación mayo / 2017) - Unigloves (Curitiba, PR Brasil); Grupo 4 - Azul (lote Z0416417C con fecha de fabricación marzo / 2017) Unigloves (Curitiba, PR - Brasil); Grupo 5 - Transparente (Lote SVFBAA005M con fecha de fabricación enero / 2018) Descarpack (São Paulo, SP - Brasil).

Se retiraron los guantes del lateral de la caja, para que el investigador no sufriera deformación alguna. Fueron sometidos a una prueba de inspección visual para descartar aquellos que tuvieran regiones adheridas, agujeros o desgarros visibles a simple vista, sin embargo, si ocurrieran quedarían registrados.

Todos los guantes se llenaron con $700 \mathrm{ml}$ de agua filtrada y 2 gotas de colorante violeta de genciana al $1 \%$ ya mezclado en un recipiente de plástico a temperatura ambiente. Esta proporción se basó en el estudio de 1999 de Bezerra y Pinheiro, ${ }^{1}$ ya que no provocó cambios en la expansión de los guantes. Inmediatamente después del llenado, se amarraron en la parte superior correspondiente al mango, con hilo de algodón $85 \%$, tipo $4 / 4$ y luego se secaron con toallas de papel absorbente y se colgaron con el hilo mismo sobre el soporte, equidistantes entre sí por $20 \mathrm{~cm}$, en un de 1 metro de altura del suelo, previamente recubierto con papel metro blanco y encima de éste, papel absorbente, por un período de 2 horas. Cada 30 minutos, el investigador evaluó si había algún cambio en su permeabilidad y si la toalla de papel absorbente mostraba algún mojado. El ambiente en el que se realizó la prueba, presentó condiciones favorables para la investigación, siendo lugar cerrado, sin cambios de calor y viento, para no provocar transpiración en los guantes o que el viento pudiera desviar el recorrido de la gota enmascarando el lugar correspondiente a cada guante.

La clasificación del nivel de permeabilidad fue la misma que la adoptada por Batista et al 2016 [5]. Descrito en tres tipos diferentes: Grado 0 - si no hay fuga; Grado 1: si el guante está húmedo; Grado 2: si gotea. Esta diferencia clasifica la permeabilidad del guante. Se realizaron 4 registros en el formulario con intervalos de 30 minutos, totalizando 2 horas.

El análisis estadístico se realizó con base en la prueba $\mathrm{Chi}^{2}$ con un nivel de significación estadística del 5\%.

\section{Resultados}

Los guantes analizados no mostraron perforaciones, desgarros ni regiones adheridas. Así, el 100\% de los guantes que se quitaron al azar eran aptos para su uso en la investigación. 
Tabla 1: Aparición de la permeabilidad de los guantes en 90 minutos. Salvador-Bahía, 2018

\begin{tabular}{|c|c|c|c|c|c|c|}
\hline Variables & $\begin{array}{c}\text { Blanca } \\
\text { Unigloves } \\
\text { n (\%) }\end{array}$ & $\begin{array}{c}\text { Rosa } \\
\text { Unigloves } \\
\text { n (\%) }\end{array}$ & $\begin{array}{c}\text { Negro } \\
\text { Unigloves } \\
\text { n (\%) }\end{array}$ & $\begin{array}{c}\text { Azules } \\
\text { Unigloves } \\
\text { n (\%) }\end{array}$ & $\begin{array}{c}\text { Transparentes } \\
\text { Descarpack } \\
\text { n (\%) }\end{array}$ & Valor de $\mathrm{p}$ \\
\hline \multicolumn{6}{|c|}{ Grado de Permeabilidad } & $* 0,548$ \\
\hline Ausencia & $19(95 \%)$ & $20(100 \%)$ & $20(100 \%)$ & $20(100 \%)$ & $19(95 \%)$ & \\
\hline Humedad & $1(5 \%)$ & 0 & 0 & 0 & $1(5 \%)$ & \\
\hline
\end{tabular}

* Prueba de chi-cuadrado. $p<0,05$.

En los tiempos de 30 y 60 minutos, los guantes en los diferentes colores tuvieron un comportamiento similar y todos no cambiaron en relación a la humedad y el goteo.

Los resultados para los tiempos de
90 y 120 minutos en relación al grado de permeabilidad se describen en las tablas 1 y 2 . Se puede observar que, independientemente del color y el tiempo de exposición, no se encontraron diferencias estadísticas entre los grupos evaluados.

Tabla 2: Ocurrencia de la permeabilidad del guante en 120 minutos. Salvador-Bahía, 2018.

\begin{tabular}{|l|c|c|c|c|c|c|}
\hline Variables & $\begin{array}{c}\text { Blanca } \\
\text { Unigloves } \\
\mathrm{n}(\%)\end{array}$ & $\begin{array}{c}\text { Rosa } \\
\text { Unigloves } \\
\mathrm{n}(\%)\end{array}$ & $\begin{array}{c}\text { Negro } \\
\text { Unigloves } \\
\mathrm{n}(\%)\end{array}$ & $\begin{array}{c}\text { Azules } \\
\text { Unigloves } \\
\mathrm{n}(\%)\end{array}$ & $\begin{array}{c}\text { Transparentes } \\
\text { Descarpack } \\
\mathrm{n}(\%)\end{array}$ & Valor de p \\
\hline Grado de Permeabilidad & \multirow{7}{|l|}{${ }^{\circ} 0,746$} \\
\hline Ausencia & $18(90 \%)$ & $19(95 \%)$ & $20(100 \%)$ & $19(95 \%)$ & $19(95 \%)$ & \\
\hline Humedad & $1(5 \%)$ & $1(5 \%)$ & 0 & $1(5 \%)$ & 0 & \\
\hline Goteo & $1(5 \%)$ & 0 & 0 & 0 & $1(5 \%)$ & \\
\hline
\end{tabular}

* Prueba de chi-cuadrado. $\mathrm{p}<0,05$.

\section{Discusión}

Se buscó, a lo largo de este estudio, evaluar la permeabilidad de los guantes de procedimiento y si sus diferentes colores o material de fabricación podrían influir en la permeabilidad, o incluso exponer al dentista a la contaminación.
Los guantes para procedimientos no quirúrgicos son productos fabricados con caucho natural, caucho sintético o mezclas de caucho natural y sintético, de un solo uso, para uso en procedimientos no quirúrgicos en el cuidado de la salud. ${ }^{6}$

La elección de los guantes fue aleatoria, 
analizada visualmente antes y después de ser llenado con una solución específica para la investigación. Los resultados y metodología de la presente investigación son similares a los de Batista et $a l^{5}$ que demuestran que los guantes de procedimiento muestran permeabilidad a las pocas horas de trabajo y en algunos casos pueden presentar mayor grado de humedad o incluso goteo.

En la investigación de Batista et $a l^{5}$ se evaluaron 10 cajas de guantes de procedimiento de látex y se estudiaron 1.007 guantes ambidiestros, de los cuales el 3,87\% reprobó la prueba de inspección visual y los guantes adecuados para la prueba de permeabilidad fallaron, diecinueve guantes $(1,96 \%)$ con grado 1 o 2 según la escala mencionada anteriormente, en la investigación en boga se obtuvo mayor porcentaje de humedad en blanco (10\%), en rosa (5\%), en azul (5\%) y guantes transparentes (5\%). El goteo resulta solo en guantes blancos (5\%) y guantes transparentes (5\%).

Sin embargo, al comparar estos datos con el trabajo de Lopes, queda claro la concordancia en presencia de agujeros visibles que mancharían el papel donde solo el 5\% de los guantes probados presentaban esta falla, pero hubo una fuerte discrepancia en cuanto a humedad, donde presenta el $100 \%$. de humedad en los guantes probados después de 2 horas de prueba.

Como resultado, los guantes de diferentes colores o materiales no afectan significativamente la seguridad del profesional. El mayor riesgo de uso es la falta de evaluación de las condiciones en las que se encuentran los guantes. La atención al tiempo de servicio es otro factor a considerar, una vez que se utiliza correctamente y se cambia cada dos horas de uso.

\section{Conclusión}

Aún con la limitación de la metodología adoptada en el presente trabajo, se puede concluir que los guantes ensayados no presentaron diferencias en relación a la humedad y al goteo, pudiendo cualquiera de ellos estar indicado para uso profesional en procedimientos no quirúrgicos, siempre que se cumplan las normas y el tiempo máximo de dos horas.

\section{Conflictos de interés}

Los autores informan que no existen conflictos de intereses en este trabajo.

\section{Aspectos éticos}

El presente estudio no utilizó una base de datos, ni utilizó animales y/o humanos.

\section{Referencias bibliográficas}

1. Bezerra, SRS; Pinheiro, JT. Avaliação da integridade das luvas de procedimentos utilizadas na clínica endodôntica. R. Cons. Reg. Odontol. Pernamb., Recife, v.2,n.2, p.95-101, out. 1999.

2. Serratine ACP, Pacheco E, Miero M. Avaliação da integridade das luvas cirúrgicas após a utilização em cirurgias odontológicas. Arquivos Catarinenses de Medicina. 2007;36(1):85-9. 
3. Cavalcanti AL, Costa LB, Dantas OAB, Melo ES. Avaliação da integridade de luvas de procedimentos após atendimento odontológico. POS - Perspect. Oral Sci. 2010;2(2):15-9.

4. Lopes N, Prates N, Rabelo R, Cruz JFW. Análise da permeabilidade das luvas de látex para procedimento mais utilizados por alunos da faculdade de Odontologia da unidade federal da Bahia. Rev. CI. Biol. 2009; 8(2): 2916-12.

5. Batista JNS, Rodrigues MDRA, Gomes RMV, Rodrigues CDT, Gonçalves AR. Avaliação da qualidade de luvas de látex utilizadas em procedimentos odontológicos. Rev Bras Odontol. 2016; 73(2): 107-11.

6. Agência Nacional de Vigilância Sanitária (Brasil). Resolução RDC no . 5, de 15 de fevereiro de 2008. Diário Oficial [da] República Federativa do Brasil, Poder Executivo, Brasília, DF, 18 fev. 2008.

Recibido: $24 / 07 / 2020$

Aceptado: 19/12/2020

Correspondencia: Janaina Damasceno, correo: janaina.damasceno.santos@gmail.com 